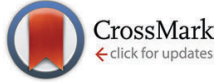

Cite this: Phys. Chem. Chem. Phys., 2015, 17, 19643

Received 15th April 2015, Accepted 29th June 2015

DOI: $10.1039 / c 5 c p 02193 f$

www.rsc.org/pccp

\section{Laser-driven electron dynamics for circular dichroism in mass spectrometry: from one- photon excitations to multiphoton ionization}

\author{
Dominik Kröner
}

\begin{abstract}
The distinction of enantiomers is a key aspect of chemical analysis. In mass spectrometry the distinction of enantiomers has been achieved by ionizing the sample with circularly polarized laser pulses and comparing the ion yields for light of opposite handedness. While resonant excitation conditions are expected to be most efficient, they are not required for the detection of a circular dichroism (CD) in the ion yield. However, the prediction of the size and sign of the circular dichroism becomes challenging if non-resonant multiphoton excitations are used to ionize the sample. Employing femtosecond laser pulses to drive electron wavepacket dynamics based on ab initio calculations, we attempt to reveal underlying mechanisms that determine the CD under non-resonant excitation conditions. Simulations were done for $(R)$-1,2-propylene oxide, using time-dependent configuration interaction singles with perturbative doubles (TD-CIS(D)) and the aug-cc-pVTZ basis set. Interactions between the electric field and the electric dipole and quadrupole as well as between the magnetic field and the magnetic dipole were explicitly accounted for. The ion yield was determined by treating states above the ionization potential as either stationary or non-stationary with energy-dependent lifetimes based on an approved heuristic approach. The observed population dynamics do not allow for a simple interpretation, because of highly non-linear interactions. Still, the various transition pathways are governed by resonant enantiospecific $\mathrm{n}$-photon excitation, with preferably high transition dipole moments, which eventually dominate the $C D$ in the ionized population.
\end{abstract}

\section{Introduction}

Mass spectrometry (MS) is a widely used and exceptionally sensitive analytic technique. While the obtained fragmentation pattern carries structural information, the distinction of isomers can become challenging, such that MS is often combined with other analytical, e.g. chromatographic, techniques or applied in a tandem setup of sequential mass analyzers. However, shaped femtosecond laser pulses, optimized in computer assisted feedback loops, ${ }^{1,2}$ may be used to identify constitutional isomers and even quantify their ratio in a mixture. ${ }^{3}$ When it comes to enantiomers, i.e. non-superposable molecules of the same constitution and related by reflection, the fragmentation pattern cannot simply be consulted to distinguish them. If, however, circular polarized nanosecond pulses are used for the ionization of the molecules, a difference in the ion yield is found, depending on the handedness of the light and the sample. ${ }^{4,5}$ Accordingly, a circular dichroism in the ion yield can be determined by

Chemistry Department - Theoretical Chemistry, Universität Potsdam,

Karl-Liebknecht-Straße 24-25, D-14476 Potsdam, Germany.

E-mail: Dominik.Kroener@uni-potsdam.de comparing the ion yields of two measurements with opposite circular polarization, similar to the experimental proof of enantioselective laser pulse control proposed by us a few years before. ${ }^{6,7}$

Circular dichroism in ion yields can also be detected employing shaped circular polarized femtosecond (fs) laser pulses. ${ }^{8}$ Despite the advances, the optimal conditions and laser pulse parameters for the maximum chiral distinction of enantiomers in MS remain unknown. While resonance enhanced multiphoton ionization (REMPI) is usually employed, Horsch et al. measured the circular dichroism (CD) in ion yields for $(R)$-propylene oxide after nonresonant multiphoton ionization with fs-laser pulses. ${ }^{9}$ Different laser frequencies were tested which were significantly lower than the transition frequency to the first electronic exited state. Measurements of the ion yields with respect to the laser pulse energy indicated three- to seven-photon processes for the different excitation wavelengths. Consequently, m-photon transitions composed of a resonant n-photon excitation to an electronic excited states followed by an (m-n)-photon transition to the ionization continuum were assumed. However, the detected $\mathrm{CD}$ in the ion yields did not correspond to the CD (or anisotropy factor) in one-photon absorption at the respective n-photon frequency. A fully satisfactory assignment of the excitation 
wavelengths to resonant n-photon transitions did, hence, not succeed, leaving the explanation for the origin of the detected $\mathrm{CD}$ in the ion yield incomplete.

For $(R)$-3-methylcyclopentanone we have proven that the CD in the ion yield after REMPI can be predicted by laser-driven quantum electron dynamics based on $a b$ initio calculations, namely time-dependent configuration interaction singles with perturbative doubles (TD-CIS(D)). ${ }^{10,11}$ In particular, the impact of the laser pulse duration was successfully reproduced by our approach, allowing for an explanation of the observed decrease of the CD for very short laser pulses. ${ }^{10}$ The requirement was a multi-state system based on ab initio data with electric and magnetic dipole transition elements between every electronic state to allow for a dynamic polarization of the molecule with the laser. Although our approach allowed for the investigation of the CD as a function of various laser pulse parameters so far, ${ }^{11}$ it required an initial resonant transition to a specific electronic excited state.

In order to simulate the experiments by Horsch et al.,, 12 applying non-resonant multiphoton ionization, we, hence, have to extend our model by several aspects. We will demonstrate ways for the calculation of the CD based on stationary or nonstationary electronic states above the ionization potential. Moreover, electric quadrupole interactions will be introduced into the interaction Hamiltonian, and their contribution to resonant and non-resonant multiphoton excitations will be studied. Based on these extensions we are able to clarify which (enantiospecific) transitions are responsible for the $\mathrm{CD}$ in the ion yield after nonresonant laser pulse ionization.

Note that, our approach differs in several ways from the one of Ma and Salam, who studied the related problem of optimal selective excitation of enantiomers in a racemic mixture by circular polarized laser pulses. ${ }^{13}$ Their investigations were based on the analytical description of a two-level model system, for which resonant and off-resonant one-photon absorptions, mediated by electric and magnetic dipole interactions, were compared showing that off-resonant conditions significantly decreased the enantioselectivity.

The paper is organized as follows: Section 2 describes the model system, the applied quantum chemical methods and the extensions of the laser-driven many electron electron dynamics for the simulation of the circular dichroism in non-resonant multiphoton excitations. In Section 3 the results of the ab initio calculations as well as of the quantum dynamics are presented and discussed. Conclusions are given in Section 4. Appendices A to E give more insight into the quality of the $a b$ initio results, and more detailed explanations for the more complex findings, partly based on a two-level model system.

\section{Model and theory}

\subsection{Model system}

The molecule investigated in mass spectrometry is 1,2-propylene oxide, ${ }^{9}$ also known as epoxypropane. As in the experiment, we focus on the $(R)$-enantiomer, which will be abbreviated by

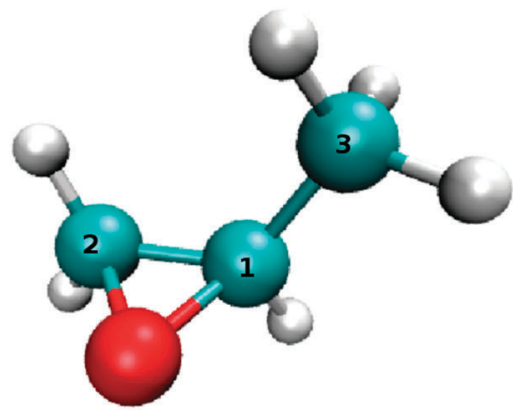

Fig. 1 Minimum energy geometry of (R)-propylene oxide, MP2/aug-cc-pVTZ.

$(R)$-PO in the following. The minimum energy geometry was obtained from MP2 (2nd order Møller-Plesset perturbation theory) using the triple-zeta augmented correlation-consistent basis set aug-cc-pVTZ. ${ }^{14-16}$ The optimized structure is shown in Fig. 1. Characteristic geometric parameters are given in Table 6 in Appendix B. Simulated infrared (IR) and vibrational CD (VCD) are presented in Appendix C. The agreement with available experimental data is good.

Next, vertical excitation energies to the first $n=149$ singlet electronic excited states were computed for the minimum energy geometry with CIS(D) (configuration interaction singles with perturbative doubles correction) for the aug-cc-pVTZ basis set. ${ }^{17}$ The computed energies are later used for the electron wavepacket dynamics, see Section 2.2, and to simulate UV and electronic CD (ECD) spectra for comparison with experimental spectra. For the ECD spectrum the rotatory strengths $R_{j}$ for the transition from the electronic ground state $S_{0}$ to the $j$ th electronic excited state $S_{j}$ are needed additionally. The rotatory strength is defined as

$$
R_{j}=\Im\left\{\left\langle\Phi_{0}|\widehat{\vec{\mu}}| \Phi_{j}\right\rangle \cdot\left\langle\Phi_{j}|\widehat{\vec{m}}| \Phi_{0}\right\rangle\right\}
$$

with $\Phi_{j}$ being the electronic wavefunction of the $j$ th state. $\widehat{\vec{\mu}}=\sum_{k} q_{k} \vec{r}_{k}$ and $\widehat{\vec{m}}=\sum_{k} \frac{q_{k}}{2 m_{k}}\left(\vec{r}_{k} \times \widehat{\vec{p}}_{k}\right)$ are the electric and magnetic dipole operators for masses $m_{k}$ with charges $q_{k}$ at positions $\vec{r}_{k}$ and with momenta $\hat{\vec{p}}_{k}=-i \hbar \vec{\nabla}_{k}$ (note that, the nuclei are fixed). The required electric and magnetic transition dipole moments between electronic ground and excited states are provided by the CIS calculations. The Gaussian broadened UV and ECD spectra are calculated as described in ref. 11.

For the quantum electron dynamics electric, $\vec{\mu}_{i j}=\left\langle\Phi_{i}|\widehat{\vec{\mu}}| \Phi_{j}\right\rangle$, and magnetic transition dipole moments, $\vec{m}_{i j}=\left\langle\Phi_{i}|\widehat{\vec{m}}| \Phi_{j}\right\rangle$, between every pair of electronic states $i$ and $j$ have to be considered. ${ }^{18}$ They are also provided by the CIS calculations. Moreover, permanent electric dipole (and quadrupole) moments of each electronic state, $\vec{\mu}_{i i}$, were obtained from additional CIS calculations based on the generalized electronic density. ${ }^{19}$ Note that all $\vec{m}_{i i}$ are zero, and $\vec{m}_{i j}=-\vec{m}_{j i}=\vec{m}_{j i}{ }^{*}$ due to the (angular) momentum operator in $\widehat{\vec{m}}$. As the electric transition quadrupole moments are often in the same order of magnitude as the magnetic transition dipole moments, they are considered as 
well in the wavepacket dynamics. Additional CIS calculations based on the transition density matrices between every electronic state $i$ and $j$ yielded all required electric transition quadrupole moment tensors $\underline{\underline{Q}}_{i j}=\left\langle\Phi_{i}|\underline{\underline{Q}}| \Phi_{j}\right\rangle$, where $\hat{Q}_{\alpha \beta}=\sum_{k} q_{k} \vec{r}_{k, \alpha} \vec{r}_{k, \beta}$ is the $\alpha \beta$-Cartesian component $(\alpha, \beta \in\{x, y, z\})$ of the electric quadrupole operator. ${ }^{18}$ Since the lower order moment of the molecule, i.e. the electric dipole is non-zero, the electric quadrupole moment depends on the choice of the origin of the coordinate system. Moreover, an origin-dependence exists for the magnetic transition dipole moments, too. The center of charge was chosen as the origin. All $a b$ initio calculation were done with the Gaussian09 program package. ${ }^{20}$

\subsection{Electron wavepacket dynamics}

The electron dynamics are simulated in the state representation, using the time-dependent configuration interaction singles method, with perturbative doubles (TD-CIS(D)) treatment for the energies of the electronic states, as described elsewhere. ${ }^{21,22}$ In total $n=150$ electronic states were used if not stated otherwise. The propagation was carried out employing the Runge-Kutta method of fourth order with a time step of $\Delta t=2.5$ attoseconds, see Table 1 .

The interaction with the laser is expressed as: ${ }^{23,24}$

$$
\hat{V}(t)=-\frac{1}{2} \mathcal{E}_{0} s(t)\left[\left(\widehat{\vec{D}} \widehat{\vec{e}}_{\delta}\right)^{\dagger} \mathrm{e}^{i \omega\left(t-t_{\mathrm{c}}\right)}+\left(\widehat{\vec{D}} \widehat{\vec{e}}_{\delta}\right) \mathrm{e}^{-i \omega\left(t-t_{\mathrm{c}}\right)}\right]
$$

with $\widehat{\vec{D}}=\widehat{\vec{\mu}}-\frac{1}{c} \widehat{\overrightarrow{e_{z}}} \times \widehat{\vec{m}}+\frac{1}{2} i \underline{\underline{Q}} \vec{k}$, where the wavevector $\vec{k}=\frac{\omega_{\widehat{\vec{e}}}}{c}$ for the field propagating in $z$-direction $\left(\widehat{\vec{e}}_{z}\right.$ is the unit vector in $z$-direction), see below. In contrast to ref. 11 we now include

Table 1 Standard parameters for most simulations ( $n$ : number of electronic states, $\Delta t$ : time step, $N$ : number of orientations, $d$ : escape distance) and typical laser pulse parameters for one-, two- and four-photon $S_{0} \rightarrow S_{2}$ excitations in $(R)$-PO. See text for details

\begin{tabular}{|c|c|}
\hline Parameter & Value \\
\hline$n$ & 150 \\
\hline$\Delta t[\mathrm{as}]$ & 2.5 \\
\hline$N$ & 144 \\
\hline$d\left[a_{0}\right]$ & 100 \\
\hline$t_{\mathrm{p}}[\mathrm{fs}]$ & 200 \\
\hline fwhm $_{\mathrm{I}}[\mathrm{fs}]$ & 73 \\
\hline$\omega_{02}\left[\mathrm{eV} \hbar^{-1}\right]$ & 7.01 \\
\hline $\mathcal{E}_{0}\left[\mathrm{GV} \mathrm{m}^{-1}\right]$ & 0.1 \\
\hline $\bar{I}\left[\mathrm{GW} \mathrm{cm} \mathrm{cm}^{-2}\right]$ & 0.50 \\
\hline$\gamma_{\mathrm{K}}$ & 1150 \\
\hline$\omega_{02} / 2\left[\mathrm{eV} \hbar^{-1}\right]$ & 3.50 \\
\hline $\mathcal{E}_{0}\left[\mathrm{GV} \mathrm{m} \mathrm{m}^{-1}\right]$ & 1.0 \\
\hline $\bar{I}\left[\mathrm{GW} \mathrm{cm}^{-2}\right]$ & 50 \\
\hline$\gamma_{\mathrm{K}}$ & 57.4 \\
\hline$\omega_{02} / 4\left[\mathrm{eV} \hbar^{-1}\right]$ & 1.75 \\
\hline $\mathcal{E}_{0}\left[\mathrm{GV} \mathrm{m}^{-1}\right]$ & 10 \\
\hline $\bar{I}\left[\mathrm{TW} \mathrm{cm}^{-2}\right]$ & 5.0 \\
\hline$\gamma_{\mathrm{K}}$ & 2.87 \\
\hline
\end{tabular}

electric quadrupole interactions in addition to the magnetic dipole interactions. The electric field of the laser is given by:

$$
\overrightarrow{\mathcal{E}}(t)=\frac{1}{2} \mathcal{E}_{0} s(t)\left[\widehat{\vec{e}}_{\delta}^{\dagger} \mathrm{e}^{i \omega\left(t-t_{\mathrm{c}}\right)}+{\widehat{\overrightarrow{e_{\delta}}}}_{\delta} \mathrm{e}^{-i \omega\left(t-t_{\mathrm{c}}\right)}\right]
$$

with the amplitude $\mathcal{E}_{0}$ and the frequency $\omega$. The pulse shape is formed by $s(t)=\cos ^{2}\left(\frac{\pi\left(t-t_{\mathrm{c}}\right)}{t_{\mathrm{p}}}\right)$ for $0 \leq t \leq t_{\mathrm{p}}$ and $t_{\mathrm{c}}=\frac{t_{\mathrm{p}}}{2}$, where the pulse duration $t_{\mathrm{p}}=2$ fwhm (full width at half maximum of the field). For better comparison with experimental conditions, the fwhm of the intensity may be calculated by: $\mathrm{fwhm}_{\mathrm{I}}=\left(1-\frac{2}{\pi} \arcsin \left(\frac{1}{\sqrt[4]{2}}\right)\right) t_{\mathrm{p}} \approx 0.364 t_{\mathrm{p}}$. The polarization is given by $\widehat{\vec{e}}_{\delta}=\frac{1}{\sqrt{2}}\left(\widehat{\overrightarrow{\vec{e}}}_{x}+\mathrm{e}^{-i \delta} \widehat{\overrightarrow{\vec{e}}}_{y}\right)$, where the phase $\delta$ may be chosen $-\frac{\pi}{2}$ for left-(LCP) and $+\frac{\pi}{2}$ for right circular polarization (RCP). The magnetic field is defined accordingly using $\overrightarrow{\mathcal{B}}=\frac{1}{c} \widehat{\vec{e}_{z}} \times \overrightarrow{\mathcal{E}}$.

The field amplitude $\mathcal{E}_{0}$ was chosen such that the impact on the system is not too strong (for reasons discussed below), while the amount of transferred population is still significantly above the numerical error, to allow for a reliable analysis of the results. Typical laser pulse parameters for one-, two- and fourphoton transitions are listed in Table 1 . The mean intensity (averaged over $t_{\mathrm{p}}$ ) is calculated as $\bar{I}=\frac{3}{16} \varepsilon_{0} c \mathcal{E}_{0}{ }^{2}$. The Keldysh parameter - defined as $\gamma_{K}=\left(\frac{\mathrm{IP}}{2 U_{\mathrm{p}}}\right)^{\frac{1}{2}}$ with $U_{\mathrm{p}}=\frac{e^{2}}{m_{\mathrm{e}}} \frac{\mathcal{E}_{0}{ }^{2}}{(2 \omega)^{2}}$ being the ponderomotive energy and the ionization potential IP = $10.22 \mathrm{eV}^{25}$ - determines the transition between two regimes of nonlinear ionization: ${ }^{26}$ multiphoton ionization (MPI) is to occur if $\gamma_{\mathrm{K}}>1$, while tunneling ionization is dominant for $\gamma \ll 1$. For all laser pulses investigate here the Keldysh parameter is well above one, see Table 1.

For (resonant) transitions to one specific electronic excited state $\mathrm{S}_{j}$, we may define the circular dichroism (CD) via the population of the excited state after laser excitation:

$$
\mathrm{CD}\left[\mathrm{S}_{j}\right]=2 \frac{P_{\mathrm{LCP}}\left[\mathrm{S}_{j}\right]-P_{\mathrm{RCP}}\left[\mathrm{S}_{j}\right]}{P_{\mathrm{LCP}}\left[\mathrm{S}_{j}\right]+P_{\mathrm{RCP}}\left[\mathrm{S}_{j}\right]}
$$

Note that the populations $P$ are either for a single molecular orientation or an average of populations of different orientations as discussed below. In contrast to previous investigations ${ }^{10,11}$ we now introduced the factor 2 in eqn (4) (and in the following CD definitions), which corresponds to the average of the sum in the denominator. This allows for a better comparability with the experiment, where the $\mathrm{CD}$ in ion yield is defined accordingly.

In our simulations the laser field interacts with the projections of $\vec{\mu}_{i j}$ and $\vec{m}_{i j}$ onto the $x-y$-plane for a given orientation of the molecule. In experiment the molecules are, however, randomly oriented. Therefore, propagations are performed for different orientations of the molecule and the results are averaged over all orientations. For it the molecule, i.e. all its (transition) dipole/quadrupole moments, is rotated around 
the laboratory fixed coordinate axes using Euler angles $\phi(\in[0,2 \pi])$ and $\theta(\in[0, \pi])$ :

$$
\begin{aligned}
& \vec{\mu}_{i j}{ }^{\prime}=\mathcal{R}_{y}(\theta) \mathcal{R}_{z}(\phi) \vec{\mu}_{i j} \\
& \vec{m}_{i j}{ }^{\prime}=\mathcal{R}_{y}(\theta) \mathcal{R}_{z}(\phi) \vec{m}_{i j} \\
& \underline{\underline{Q}}_{i j}{ }^{\prime}=\mathcal{R}_{y}(\theta) \mathcal{R}_{z}(\phi) \underline{\underline{Q}}_{i j} \mathcal{R}_{z}^{T}(\phi) \mathcal{R}_{y}^{T}(\theta)
\end{aligned}
$$

where $\mathcal{R}_{k}(\xi)$ is the rotation matrix for a rotation about the $k$-axis by $\xi$ degrees. A third rotation about the $z$-axis was omitted, because it has no influence on the laser-molecule interactions for the employed circularly polarized pulses propagating in $z$-direction and where $1 / \omega \ll t_{\mathrm{p}}$. The rotation angles are given by $\phi=i \cdot \Delta \xi$ and $\theta=j \cdot \Delta \xi$ for $i=0,1, \ldots, M-1$ or $j=0,1, \ldots, \frac{M}{2}$, where $\Delta \xi=\frac{2 \pi}{M}$. The number of considered orientations $\left(N=M \cdot\left(\frac{M}{2}+1\right)\right)$ was set to $144\left(\Delta \xi=22.5^{\circ}\right)$, if not stated otherwise. For resonant transitions to $S_{j}$ very small changes were found in the $\mathrm{CD}\left[\mathrm{S}_{j}\right]$ when increasing the number of orientations. For non-resonant excitations where practically every transition moment element may play a role, this number has to be considered a compromise between an acceptable computational effort and a reasonable degree of convergence. The few performed test calculations with a higher number of orientations did not result in important changes.

All CD values are calculated from rotationally averaged final populations (unless noted otherwise) which are obtained by: ${ }^{27}$

$$
\langle P\rangle=\frac{1}{8 \pi^{2}} \int_{0}^{2 \pi} \int_{0}^{\pi} \int_{0}^{2 \pi} P(\phi, \theta, \chi) \sin (\theta) \mathrm{d} \phi \mathrm{d} \theta \mathrm{d} \chi .
$$

In practice, populations are determined for specific molecular orientations, $P(\phi, \theta, \chi)$, and then averaged numerically according to eqn (6), however, omitting the third rotation angle $(\chi)$ and calculating the norm (now $4 \pi$ instead of $8 \pi^{2}$ ) explicitly by $\int_{0}^{2 \pi} \mathrm{d} \phi \int_{0}^{\pi} \mathrm{d} \theta \sin (\theta)$.

Note that, one can always (unless the vectors are collinear) find orientations for which the projections of $\vec{\mu}_{i j}$ and $\vec{m}_{i j}$ onto the $x y$-plane result in parallel or anti-parallel projection vectors, causing a sign change in the calculated CD-value. Therefore, rotational averaging is mandatory to balance these sign chances in the CD-values by those of all the other orientations. In addition, for a resonant excitation to an excited state $\mathrm{S}_{j}$, it is convenient to initially orient the molecule such that its interaction with the light is maximized. This is accomplished by rotating the molecule beforehand such that the corresponding electric transition dipole moment, $\mu_{0 j}$, becomes parallel to the $x$-axis, and the magnetic transition dipole moment, $m_{0 j}$, is in the $x y$-plane. That way we ensure that the target state is highly populated allowing for a reliable result for the $\operatorname{CD}\left[\mathrm{S}_{j}\right]$. Needless to say that this approach cannot uniquely be applied for nonresonant excitations with no specific target state. Moreover, a preferred orientation of the molecule could actually overestimate one specific transition moment unless rotational averaging over a huge number of orientations is carried out.
As we are first of all interested in observing circular dichroism we chose an initial orientation which is optimal for a resonant transition to $S_{2}$, because it is the state with the highest anisotropy factor within the experimental investigated range of excitations energies (6.8-9 eV), see Section 3.

\subsection{Model for ionization}

In experiment the ion yield is measured. Therefore, a determination of the $\mathrm{CD}$ is required which is more comparable to the experiment than the one used in eqn (4), in particular if the laser excitation is non-resonant. Here the challenge is not only to correctly describe the free electron, but also the transition from bound states to continuum states. The required transition matrix elements, however, cannot be obtained from our wavefunction calculations, i.e. by optimizing the neutral and cationic state, as the number of electrons differs. In addition, in the dynamics a large number of electronic eigenstates also above the IP is required to cover a maximal portion of the dynamic polarizability of the molecule. ${ }^{28}$ Hence, electronic states above the IP, as obtained from the $a b$ initio calculations, cannot simply be omitted or exchanged by a continuum. Consequently, the population of these, quasi-ionized, states above the IP can be used to quantify the extent of ionization.

Two models for the detection of the ionization yield are used in this work. Both rely on the sum of the population of all eigenstates above the IP, obtained from the total population remaining below the IP by:

$$
P\left[\mathrm{~S}_{>\mathrm{IP}}\right]=1-\sum_{i<\mathrm{IP}} P\left[\mathrm{~S}_{i}\right] .
$$

In practice, the sum in eqn (7) covers the populations of states $\mathrm{S}_{0}$ to $\mathrm{S}_{64}$, as we find 65 electronic states below the IP (and $150-$ $65=85$ states above the IP). The vast majority of these states is dominated by electronic excitations to Rydberg like orbitals; their large number is promoted by the diffuse functions of the basis set. Now the IP is not given by its experimental value $(10.22 \mathrm{eV})$, but by the one consistent with our $a b$ initio calculations $(11.81 \mathrm{eV})$, i.e. the negative orbital energy of the HOMO (highest occupied molecular orbital) according to Koopmans' theorem.

The quasi ionized population in eqn (7) can be used to calculate the $\mathrm{CD}[>\mathrm{IP}]$ after the interaction with the laser pulse:

$$
\mathrm{CD}[>\mathrm{IP}]=2 \frac{P_{\mathrm{LCP}}\left[\mathrm{S}_{>\mathrm{IP}}\right]-P_{\mathrm{RCP}}\left[\mathrm{S}_{>\mathrm{IP}}\right]}{P_{\mathrm{LCP}}\left[\mathrm{S}_{>\mathrm{IP}}\right]+P_{\mathrm{RCP}}\left[\mathrm{S}_{>\mathrm{IP}}\right]} .
$$

This ansatz may, however, cause artefacts in the $\mathrm{CD}[>\mathrm{IP}]$, if the population enriched in the states above the IP is transferred back to energetically lower states. To minimize this effect, the field intensity should be kept low. Most certainly one can also try to minimize the effect by increasing the number of basis functions. However, this will not only increase the computational effort of the ab initio calculations, but most notably of the quantum dynamics simulation. Another problem is that the transitions to states above the IP may also be enantiospecific in this model. But in experiment there are no indications that the ionization step has an effect on the CD in the ion yield. ${ }^{29}$ 
Therefore, in order to reduce the effects of enantiospecific excitations to "ionized" states and population back-transfer, we can alternatively make the electronic states above the IP $(11.81 \mathrm{eV})$ non-stationary by replacing their real eigenenergies, $E_{n}(\geq \mathrm{IP})$, by complex ones: $E_{n}-i \hbar \gamma_{n} / 2$. Afterward the molecular Hamiltonian will not be Hermitian any more and, as such, the time evolution will not be unitary any more, i.e. the population of these states will decay irreversibly. The are many ways, how the decay rates $\gamma_{n}$ may be chosen. In a heuristic model that proved to be suitable for the description of ionization in TD-CIS calculations the rates were determined by: ${ }^{28}$

$$
\gamma_{n}=\sum_{a} \sum_{r}\left|D_{a, n}^{r}\right|^{2} \frac{\sqrt{2 \varepsilon_{r} / m_{\mathrm{e}}}}{d}
$$

for $\varepsilon_{r}>0$. The model assumes an escape velocity of the electron of $v=\sqrt{2 \varepsilon_{r} / m_{\mathrm{e}}}=\frac{d}{\tau}$, where $\varepsilon_{r}$ is the energy of the orbital from which the electron escapes, and $d$ is the escape distance. The rates are weighted by the probability of the excitation which is given by the absolute squared value of the corresponding CI-coefficients, $D_{a, n}^{r}$, for an electron excitation from the occupied orbital $a$ to the virtual orbital $r$. Hence, the lifetime of the states above the IP are given by $\tau_{n}=\frac{1}{\gamma_{n}}$; those below the IP are infinite. According to eqn (9), the higher the orbital energy of a virtual orbital the lower the lifetime of an electronic state which is dominated by this orbital. Orbital energies and CI-coefficients are obtained from the CIS calculations. ${ }^{18}$ The parameter $d$ may be used to scale the rates. In contrast to studies by others, ${ }^{28}$ we wish to adjust the rates such that a meaningful comparability to the $\mathrm{CD}[>\mathrm{IP}]$ is ensured, but preventing the return of population to states below the IP. However, too high rates may suppress transient population of states above the IP which has an impact on the CD as well, see Discussion in Section 3.4. Therefore, a rather high value of $100 a_{0}$ was chosen for $d$.

Worth mentioning are a similar approaches to model ionization in TD-CIS which are based on complex absorbing potentials (CAP) with space-grid or atom-centered basis functions, see e.g. ref. 30 and 31 .

The $\mathrm{CD}$ in case of non-stationary states above the IP is still calculated using eqn (7) and (8), but will be denoted $\mathrm{CD}_{\text {ion }}$ to tell it apart from the $\mathrm{CD}[>\mathrm{IP}]$. Note that for the $\mathrm{CD}_{\text {ion }}$, however, $P\left[\mathrm{~S}_{>\mathrm{IP}}\right]$ covers the decayed as well as the remaining population above the IP (if existing). In both approaches, $P\left[\mathbf{S}_{>\mathrm{IP}}\right]$ may be considered a lower limit for the "ionized" population, as a large number of states are missing to the (quasi) continuous basis set limit.

\section{Results}

\subsection{UV and ECD spectra}

The simulated UV and ECD spectra are shown in Fig. 2. The spectra are based on the vertical excitation energies, oscillator strengths or rotatory strengths, see eqn (1), calculated with CIS(D)/aug-cc-pVTZ. Selected values are given in Table 2. At first

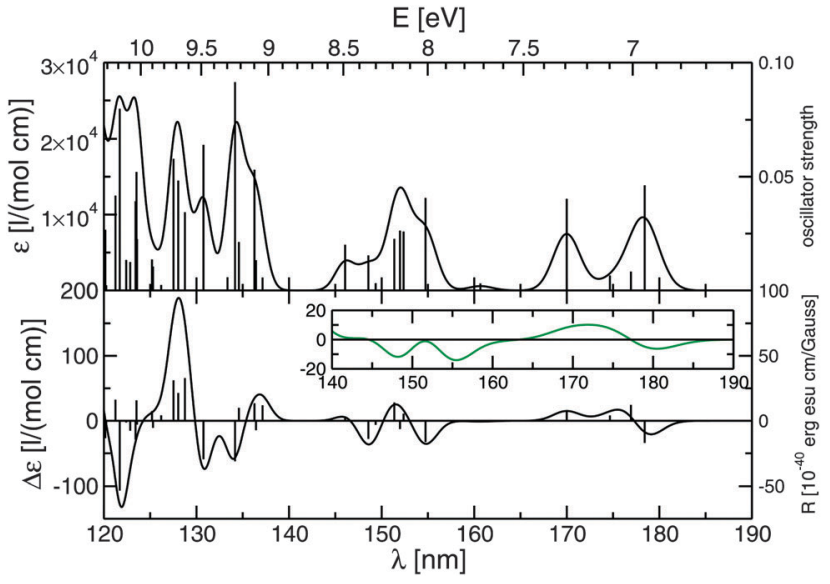

Fig. 2 Simulated UV (top) and ECD (bottom) spectra of of (R)-propylene oxide, as obtained from CIS(D)/aug-cc-pVTZ. Gaussian broadening with $500 \mathrm{~cm}^{-1}(\hat{=} \mathrm{HWHM} / \sqrt{2 \ln 2})$. Oscillator strengths or rotatory strengths are plotted as stick spectra. Spectra are shown up to ionization potential of $10.22 \mathrm{eV}^{25}$ Inset show the ECD spectrum with a Gaussian broadening of $1000 \mathrm{~cm}^{-1}$ for an easier comparison with the experimental gas phase spectrum, see text and ref. 32 and 33 .

glance three main peaks are found between $185 \mathrm{~nm}(6.7 \mathrm{eV})$ and $140 \mathrm{~nm}(8.9 \mathrm{eV})$ in agreement with experimental observations. ${ }^{32}$ Some of the bands result however from several electronic states, some of which are close in energy, with strongly varying oscillator strengths, see Table 2 . This and the fact that the gas phase spectrum shows a fine structure attributable to vibrational modes (which are not considered in our model) make a straight comparison with experiment rather complicated.

Although agreement with experiment is found for the most part, our results deviate from experimental one-photon absorption spectra as well as from theoretical data based on coupled cluster (CC) or symmetry-adapted cluster-CI (SAC-CI) calculations in some parts. ${ }^{34,35}$ These deviations are not surprising given the quality of the CIS(D) method and the fact that most excited states are of Rydberg character which is difficult to capture entirely. The simulated UV and ECD spectra are analysed in more detail in Appendix A. The analysis shows that CIS(D) provides a reasonable description of the electronic structure, in particular

Table 2 Selection of the energetically lowest excited state energies $E$, corresponding wavelengths $\lambda$, oscillator strengths $f^{\text {osc }}$, rotatory strengths $R$ (in $10^{-40}$ erg esu $\mathrm{cm}$ per Gauss), and main character of the electronic transition, as obtained from CIS(D)/aug-cc-pVTZ

\begin{tabular}{llllcl}
\hline $\mathrm{S}_{j}$ & $E_{j}[\mathrm{eV}]$ & $\lambda_{j}[\mathrm{~nm}]$ & $f_{j}^{\text {osc }}$ & $R_{j}$ & Main character \\
\hline $\mathrm{S}_{1}$ & 6.95 & 178 & 0.0462 & -17.0 & $\mathrm{n} \rightarrow$ Ryd $(3 \mathrm{~s})$ \\
$\mathrm{S}_{2}$ & 7.01 & 177 & 0.0083 & 12.3 & $\mathrm{n} \rightarrow$ Ryd $(3 \mathrm{~s})$ \\
$\mathrm{S}_{3}$ & 7.10 & 175 & 0.0066 & 4.16 & $\mathrm{n} \rightarrow$ Ryd $(3 \mathrm{sp})$ \\
$\mathrm{S}_{4}$ & 7.29 & 170 & 0.0403 & 7.37 & $\mathrm{n} \rightarrow$ Ryd $(3 \mathrm{p})$ \\
$\mathrm{S}_{5}$ & 7.72 & 161 & 0.0031 & -0.521 & $\sigma \rightarrow$ Ryd $(3 \mathrm{~s})$ \\
$\mathrm{S}_{6}$ & 8.01 & 155 & 0.0407 & -16.3 & $\sigma \rightarrow$ Ryd $(3 \mathrm{sp})$ \\
$\mathrm{S}_{7}$ & 8.14 & 152 & 0.0259 & 5.53 & $\mathrm{n} \rightarrow$ Ryd $\left(3 \mathrm{p}^{\prime}, 3 \mathrm{~s}^{\prime \prime}\right)$ \\
$\mathrm{S}_{8}$ & 8.16 & 152 & 0.0263 & -6.28 & $\sigma \rightarrow$ Ryd $\left(3 \mathrm{~s}^{\prime}\right)$ \\
$\mathrm{S}_{9}$ & 8.19 & 151 & 0.0227 & 14.1 & $\mathrm{n} \rightarrow \operatorname{Ryd}\left(3 \mathrm{p}^{\prime \prime}\right)$ \\
$\mathrm{S}_{10}$ & 8.30 & 149 & 0.0032 & -3.08 & $\mathrm{n} \rightarrow \operatorname{Ryd}\left(3 \mathrm{~s}^{\prime \prime \prime}\right)$ \\
$\mathrm{S}_{11}$ & 8.35 & 149 & 0.0154 & -13.9 & $\sigma \rightarrow \operatorname{Ryd}(3 \mathrm{p})$ \\
$\mathrm{S}_{12}$ & 8.50 & 146 & 0.0201 & 3.26 & $\mathrm{n} \rightarrow \operatorname{Ryd}\left(3 \mathrm{p}^{\prime}, 3 \mathrm{~s}^{\prime \prime}\right)$
\end{tabular}


for the low lying electronic excited states. Since we wish to employ time-dependent CIS to reveal the fundamental steps of the electron dynamics, a perfect agreement with the experiment is, however, not required as the laser pulse parameter can easily be adapted to the calculated electronic structure of the system. For the following many electron dynamics the electronic structure is a good compromise between accuracy and computational effort.

\subsection{One-photon excitations}

The electron wavepacket dynamics allow comparison of resonant one- with two- or higher multi-photon excitations to a target state $\mathrm{S}_{j}$, for which the $\mathrm{CD}\left[\mathrm{S}_{j}\right]$ can easily be determine according to eqn (4). For this purpose it is important to understand which types of interactions $(\widehat{\vec{\mu}}, \widehat{\vec{m}}, \underline{\underline{Q}})$ mediate these transitions. Beforehand, a suitable target state for the analysis has to be identified. Here it is instructive to learn about the correlation between the $\mathrm{CD}\left[\mathrm{S}_{j}\right]$ and the rotatory strength or the anisotropy factor.

In a resonant one-photon transition, the difference in the population, $\Delta P\left[\mathrm{~S}_{j}\right]=P_{\mathrm{LCP}}\left[\mathrm{S}_{j}\right]-P_{\mathrm{RCP}}\left[\mathrm{S}_{j}\right]$, of the target state $\mathrm{S}_{j}$ is determined by the lengths of the corresponding electric and magnetic transition dipole vectors, $\left|\vec{\mu}_{0 j}\right|$ and $\left|\vec{m}_{j 0}\right|$, as well as the angle $\theta=\arccos \left(\frac{\Im\left(\vec{\mu}_{0 j} \cdot \vec{m}_{j 0}\right)}{\left|\vec{\mu}_{0 j}\right|\left|\vec{m}_{j 0}\right|}\right)$ between them. These values are listed in Table 3 for a selection of electronic states. Note that the angle $\theta$ is origin-dependent, as shown e.g. for computed VCD spectra. ${ }^{36}$

In a two-level system, $\Delta P$ of the target state maximizes with increasing size of the electric or magnetic transition dipole moments and for angles $\theta$ close to $0^{\circ}$ or $180^{\circ}$, as e.g. shown by Ma and Salam. ${ }^{37}$ Naturally, this seems to perfectly correspond to the rotatory strength $R_{j}=\left|\vec{\mu}_{0 j}\right|\left|\vec{m}_{j 0}\right| \cos (\theta)$, see eqn (1). While the rotational strength is determined by the product of $\left|\vec{\mu}_{0 j}\right|$ and $\left|\vec{m}_{j 0}\right|$, we observe a significant $\mathrm{CD}\left[\mathrm{S}_{j}\right]$ if $\left|\vec{m}_{j 0}\right|$ is large with

Table 3 Lengths of electric and magnetic transition dipole vectors between electronic ground and selected electronic excited states, $\vec{\mu}_{0 j}$ and $\mathfrak{I}\left(\vec{m}_{j 0}\right)$, their ratio, the angle $\theta$ between them, and the calculated anisotropy factor $g$ (CIS/aug-cc-pVTZ). Values for the $S_{0} \rightarrow S_{1}$ transition in $(R)$-3-methylcyclopentanone are given for comparison ${ }^{11}$

\begin{tabular}{|c|c|c|c|c|c|}
\hline $\mathrm{S}_{j}$ & $\left|\vec{\mu}_{0 j}\right|\left[\mathrm{e} a_{0}\right]$ & $\left|\vec{m}_{j \mathrm{o}}\right|\left[\mathrm{e} \hbar m_{\mathrm{e}}{ }^{-1}\right]$ & $\frac{\left|\vec{m}_{j 0}\right|}{\left|\vec{\mu}_{0 j}\right|}\left[a_{0} E_{\mathrm{h}} \hbar^{-1}\right]$ & $\theta\left[{ }^{\circ}\right]$ & $g\left[a_{0} E_{\mathrm{h}} \hbar^{-1}\right]$ \\
\hline $\mathrm{S}_{1}$ & 0.45 & 0.19 & 0.41 & 116 & -0.72 \\
\hline $\mathrm{S}_{2}$ & 0.19 & 0.14 & 0.76 & 11 & 3.0 \\
\hline $\mathrm{S}_{3}$ & 0.17 & 0.35 & 2.0 & 82 & 1.2 \\
\hline $\mathrm{S}_{4}$ & 0.41 & 0.085 & 0.21 & 63 & 0.38 \\
\hline $\mathrm{S}_{5}$ & 0.12 & 0.27 & 2.3 & 92 & -0.31 \\
\hline $\mathrm{S}_{6}$ & 0.42 & 0.13 & 0.32 & 128 & -0.77 \\
\hline $\mathrm{S}_{7}$ & 0.31 & 0.049 & 0.16 & 40 & 0.47 \\
\hline $\mathrm{S}_{8}$ & 0.34 & 0.17 & 0.49 & 104 & -0.47 \\
\hline $\mathrm{S}_{9}$ & 0.29 & 0.14 & 0.47 & 42 & 1.4 \\
\hline $\mathrm{S}_{10}$ & 0.11 & 0.22 & 2.0 & 105 & -2.0 \\
\hline $\mathrm{S}_{11}$ & 0.26 & 0.19 & 0.73 & 128 & -1.8 \\
\hline $\mathrm{S}_{12}$ & 0.28 & 0.16 & 0.57 & 81 & 0.36 \\
\hline $\mathrm{S}_{1}(3 \mathrm{MCP})$ & 0.029 & 0.54 & 19 & 11 & 74 \\
\hline
\end{tabular}

respect to $\left|\vec{\mu}_{0 j}\right|$. Thus, a high $\frac{\left|\vec{m}_{j 0}\right|}{\left|\vec{\mu}_{0 j}\right|}$ ratio is desired for a good chiral distinction in our simulations. Calculated values are given in Table 3. Please, recall that the $\operatorname{CD}\left[\mathrm{S}_{j}\right]$ is the weighted difference in the populations, see eqn (4); a high population of the target state (mediated by $\vec{\mu}_{0 j}$ ) is not required for a high absolute CD value. Therefore, although the absolute value of the rotational strength for the $S_{0} \rightarrow S_{1}$ excitation is quite high $\left(R_{1}=-17.0 \times 10^{-40} \mathrm{cgs}\right)$, see Table 2 , we find a $\frac{\left|\vec{m}_{j 0}\right|}{\left|\vec{\mu}_{0 j}\right|}$ ratio below one $\left(0.41 a_{0} E_{\mathrm{h}} \hbar^{-1}\right)$, see Table 3 . For the $\mathrm{S}_{0} \rightarrow \mathrm{S}_{1}$ excitation of the previously investigated $(R)-3$-methylcyclopentanone (3MCP) the $\left|\vec{m}_{j 0}\right| /\left|\vec{\mu}_{0 j}\right|$ ratio is more than forty times higher $\left(19 a_{0} E_{\mathrm{h}} \hbar^{-1}\right)$, although the rotational strength is more than two times lower in magnitude $\left(R_{0}=7.25 \times 10^{-40} \mathrm{cgs}\right){ }^{11}$ Therefore, the absolute $\mathrm{CD}\left[\mathrm{S}_{1}\right]$ value is expected to be much larger for $(R)-3 \mathrm{MCP}$ than for $(R)$-PO. The reason is that the $\mathrm{S}_{0} \rightarrow \mathrm{S}_{1}$ excitation in $3 \mathrm{MCP}$ is characterized by a $\mathrm{n} \rightarrow \pi^{*}$-transition in the carbonyl group, which is typically almost electric dipole forbidden, but strongly magnetic dipole allowed.

The $\frac{\left|\vec{m}_{j 0}\right|}{\left|\vec{\mu}_{0 j}\right|}$ ratio may be connected to the anisotropy factor $g$, which is the ratio of the $\mathrm{CD}$ to the absorption in absorption spectroscopy, i.e. the difference of the extinction coefficients for LCP and RCP $\left(\Delta \varepsilon=\varepsilon_{\mathrm{LCP}}-\varepsilon_{\mathrm{RCP}}\right)$, to the absorption: $g=\frac{\Delta \varepsilon}{\varepsilon}$. The factor may be approximated by $g=4 \frac{R_{j}}{D_{j}}=4 \frac{\left|\vec{m}_{j 0}\right|}{\left|\vec{\mu}_{0 j}\right|} \cos (\theta)$, with the electric dipole strength $D_{j}=\vec{\mu}_{0 j} \vec{\mu}_{j 0}=\left|\vec{\mu}_{0 j}\right|^{2}$, if the shapes for the CD signal and the absorption signals are the same as well as the permittivity is set to one. ${ }^{38}$ Both assumptions are reasonable, as we consider only vertical excitations in our model, and treat single molecules in vacuum. Values of $g$ are listed in Table 3. A comparison of the $g$-factors for the $\mathrm{S}_{0} \rightarrow \mathrm{S}_{1}$ transitions in $(R)-3 \mathrm{MCP}$ and $(R)$-PO supports our observations that the corresponding absolute $\operatorname{CD}\left[\mathrm{S}_{1}\right]$ values differ by about two orders of magnitude when using the same laser parameters (results not shown).

Employing TD-CIS(D), with the parameters listed in Table 1, we obtain $\mathrm{CD}\left[\mathrm{S}_{j}\right]$ values for one-photon excitations in $(R)$-PO to states $\mathrm{S}_{1}, \mathrm{~S}_{2}$ and $\mathrm{S}_{3}$ of $-0.52 \%, 2.2 \%$ and $0.87 \%$, respectively. Note that, the $\mathrm{CD}\left[\mathrm{S}_{j}\right]$ ratios almost perfectly match the ratios of the corresponding anisotropy factors, see Table 3. Moreover the values are about 137.036 times smaller than the listed $g$-values,

Table 4 Comparison of the CD[S $S_{2}$, as obtained from TD-CIS(D), for onetwo- and four-photon excitations incorporating electric dipole interactions and either magnetic dipole $(\widehat{\vec{m}})$ or electric quadrupole $(\underline{\hat{Q}})$ or both interactions for an ensemble of rotationally averaged $(R)$-PO molecules. Laser pulse parameters are given in Table 1

\begin{tabular}{cccc}
\hline $\mathrm{CD}\left[\mathrm{S}_{2}\right][\%]$ & $\omega_{02}$ & $\omega_{02} / 2$ & $\omega_{02} / 4$ \\
\hline$\widehat{\vec{m}} \neq \overrightarrow{0}, \hat{\underline{Q}}=\underline{\underline{0}}$ & 2.2 & -1.0 & 0.40 \\
$\widehat{\vec{m}}=\overrightarrow{0}, \hat{\underline{Q}} \neq \underline{\underline{0}}$ & 0.0 & -0.13 & -0.078 \\
$\widehat{\vec{m}} \neq \overrightarrow{0}, \hat{\underline{\hat{Q}}} \neq \underline{\underline{0}}$ & 2.2 & -1.2 & 0.32
\end{tabular}


because the laser interaction with the magnetic dipole is $\frac{1}{c}$ times smaller than with the electric one, see Section 2.2. Still, the anisotropy factor is not the perfect quantity to directly compare to the circular dichroism obtained after one-photon excitation, because the absolute value of $\mathrm{CD}\left[\mathrm{S}_{j}\right]$ decreases with increasing field strengths eventually ruining the almost perfect agreement with $g$, see Appendix D for more details. Only for very low field strength (weak field limit) the correlation between $\mathrm{CD}\left[\mathrm{S}_{j}\right]$ and the anisotropy factor is good.

In order to compare resonant one- and multi-photon excitations in the following, we will use the $\mathrm{S}_{0} \rightarrow \mathrm{S}_{2}$ transitions in PO, because the $|g|$-factor or $\mathrm{CD}\left[\mathrm{S}_{j}\right]$ is the highest of the low lying states.

In Table 4 we study the influence of the electric quadrupole contributions on the one-photon $\left(\omega_{02}\right) \mathrm{CD}\left[\mathrm{S}_{2}\right]$ value. If electric quadrupole interactions are included, the result will not significantly change. Very small deviations (less than $1 \%$ of the $\mathrm{CD}\left[\mathrm{S}_{2}\right]$ value) are due to the fact that perfect numerical averaging cannot be achieved here. Apparently, the $\mathrm{CD}\left[\mathrm{S}_{j}\right]$ in one-photon excitations depends only on the electric and magnetic dipole contributions. Analytically the contribution of the electric quadrupole vanishes entirely upon rotational averaging. ${ }^{23}$ For oriented molecules the electric quadrupole does, however, add to the CD-value (not shown).

In the next section, we will compare the one-photon excitation to two- and four-photon excitations.

\subsection{Multiphoton excitations}

Although $(R)$-PO is presumably non-resonantly excited before it ionizes, the slopes of the experimental parent ion yields as a function of the laser pulse energy indicate approximately three-, five- and seven-photon processes for the three different excitation wavelengths. ${ }^{9}$ Due to the manifold of electronic states, in particular when approaching the ionization potential, such resonant multiphoton excitations, i.e. where the laser frequency (within its spectral width) is an integer multiple of the respective transition frequency, are indeed possible. Therefore, for a start the $\mathrm{CD}\left[\mathrm{S}_{2}\right]$ of two- and four-photon resonant $\mathrm{S}_{0} \rightarrow \mathrm{S}_{2}$ excitations are evaluated in the following. The role of the electric quadrupole moment is in particular of interest.

In a two-photon transition the amount population transferred to the target state is significantly smaller than in the respective one-photon transition for a given field strength. The same is true when going to the four-photon excitation. In order to transfer enough population to $S_{2}$ for a reliable determination of the $\mathrm{CD}\left[\mathrm{S}_{2}\right]$, the field strength $\mathcal{E}_{0}$ is increased by a factor of ten for each doubling of the number of photons. In addition, the central laser frequency $\omega$ is half or a quarter of $\omega_{02}$. The used parameters are listed in Table 1.

Table 4 summarizes the results on (one-,) two- and fourphoton $\mathrm{S}_{0} \rightarrow \mathrm{S}_{2}$ transitions obtained from electron wavepacket dynamics. In case of the two-photon excitation the contribution of the electric quadrupole moment does not vanish upon orientation averaging and is, hence, not negligible any more. This is consistent with conclusions drawn from analytical expressions for absorption rates of elliptically polarized light, derived from time-dependent perturbation theory. ${ }^{23}$ Moreover, the absolute value of the two-photon $\mathrm{CD}\left[\mathrm{S}_{2}\right]$ is smaller than of the one-photon one; in addition, the sign has changed. This is surprising, because for a two-level system $(n=2)$ of the states $S_{0}$ and $\mathrm{S}_{2}$ (using the same pulse parameters), the two-photon $\mathrm{CD}\left[\mathrm{S}_{2}\right]$ equals $+1.9 \%$ instead of $-1.2 \%$. This is a clear indication for interaction with other electronic states. At $t_{\mathrm{p}} / 2$, i.e. when the maximum field strength is reached, population is indeed distributed among states up to $\mathrm{S}_{63}$. However, at the end of the pulse $\left(t_{\mathrm{p}}\right)$ only states $S_{0}$ to $S_{3}$ remain populated below the IP, with populations in $S_{1}$ and $S_{3}$ being negligible compared to the target states $S_{2}$. Apparently $S_{1}$ and $S_{3}$ are energetically close enough (60 to $90 \mathrm{meV}$ ), see Table 2, to partly compete with $\mathrm{S}_{2}$ due to spectral width of the (73 fs) pulse of approximately $30 \mathrm{meV}$ (3.295 $\hbar$ per fwhm $\mathrm{f}_{\mathrm{I}}$ ). Note also that the electric transition dipole moment to $S_{1}$ is more than twice the in magnitude than one to $S_{2}$. However, the interactions with the two neighboring states of $\mathrm{S}_{2}$ seem not to be enough to be alone responsible for the significant change of the $\mathrm{CD}\left[\mathrm{S}_{2}\right]$ when all 150 electronic states are included. Seemingly, transient interactions with higher states contribute to the observed CD.

In case of the four-photon excitation, the absolute value of the $\mathrm{CD}\left[\mathrm{S}_{2}\right]$ is even smaller than in the two-photon case. The reason seems to be again transient interactions, now with an even larger number of electronic states due to the lower frequency and the higher field amplitude. For a two-level system of $\mathrm{S}_{0}$ and $\mathrm{S}_{2}$ (using the same pulse parameters), we actually obtain $1.8 \%$. Moreover, the fraction of the $\mathrm{CD}\left[\mathrm{S}_{2}\right]$ value caused by electric quadrupole interactions is relatively larger for the four-photon transition $(\sim 24 \%)$ than in the case of the twophoton transition $(\sim 11 \%)$. We also note that the sign caused by the quadrupole interactions does not have to be the same as for the electric/magnetic dipole interactions only. For a correct description of the $\mathrm{CD}$ in multiphoton excitations the electric quadrupole contribution can, therefore, not be omitted. It is important to note that, in particular, the difference of the permanent electric dipole moments, $\vec{d}_{j 0}=\vec{\mu}_{j j}-\vec{\mu}_{00}$, as well as the difference between the permanent electric quadrupole moments, $\underline{\Delta}_{j 0}=\underline{\underline{Q}}_{j j}-\underline{\underline{Q}}_{00}$, should not be zero for a multiphoton excitation. ${ }^{23}$ In fact, for the limiting case of an "achiral" transition, i.e. $R_{j}=g_{j}=0, \vec{d}_{j 0}$ and $\underline{\underline{\Delta}}_{j 0}($ or $\underline{\underline{Q}})$ ) can still cause a non-zero $\mathrm{CD}\left[\mathrm{S}_{2}\right]$, as shown in Appendix E for a two-level model system.

Note that some population remains in states above the IP after the laser is off. This can be used to detect the $\mathrm{CD}[>\mathrm{IP}]$ in the following. In the next section we wish to determine the CD in the "ionized" population rather than in a target state.

\subsection{Ionization}

Since in the experiment the ion yield is detected, one of the two approaches for analyzing the "ionized" population (see Section 2.3) has to be applied if non-resonant conditions are used. In order to estimate the performance of the two models, they are tested for one-, two- and four-photon $\mathrm{S}_{0} \rightarrow \mathrm{S}_{2}$ excitations, see Table 5. We realize that the $\mathrm{CD}[>\mathrm{IP}]$ qualitatively reproduces the results of the $\mathrm{CD}\left[\mathrm{S}_{2}\right]$, but slightly differs quantitatively. In particular 
for $\omega_{02} / 2$ and $\omega_{02} / 4$ differences are not surprising, because these frequencies allow resonances to several states below the IP which can alter the CD detected above the IP.

Evidently, the result for the resonant one-photon excitation supports our previous assumptions that the detection of the CD in the population of the target state is a valid approximation for the CD in the ion yield (or at least for the "ionized" population). The small difference of the $\mathrm{CD}[>\mathrm{IP}]$ with respect to the $\mathrm{CD}\left[\mathrm{S}_{2}\right]$ could be caused by transient population transferred back to states below the IP, due to the limited number of 150 states, or simply by resonant enantiospecific transitions from $\mathrm{S}_{2}$ to states above the IP with an opposite CD effect. Indeed, for the $\mathrm{S}_{2} \rightarrow \mathrm{S}_{128}$ transition with $E_{128}-E_{2}=(7.01-14.01) \mathrm{eV}=7.00 \mathrm{eV}$, a negative $g$-value can be computed. Still, the state $S_{128}$ is not the most populated state above the IP though. This is state $S_{129}$ at $14.03 \mathrm{eV}$ due to the higher $\mathrm{S}_{2} \rightarrow \mathrm{S}_{129}$ dipole strength compared to the $S_{2} \rightarrow S_{128}$ transition. And for $S_{2} \rightarrow S_{129}$ we calculated a positive $g$-value. These findings indicate that transitions to the states above the IP may have a impact on the $\mathrm{CD}[>\mathrm{IP}]$. Yet, the effect seems to be small and should, for all we know, not appear in the ionization step.

The $\mathrm{CD}_{\text {ion }}$ results tell a somewhat different story: while for the one-photon $\mathrm{S}_{0} \rightarrow \mathrm{S}_{2}$ excitation the $\mathrm{CD}_{\text {ion }}$ is still close to the $\mathrm{CD}[>\mathrm{IP}]$ and $\mathrm{CD}\left[\mathrm{S}_{2}\right]$ values, there are significant deviations for the two- and four-photon transitions. Although the $\mathrm{CD}_{\text {ion }}$ is for all three frequencies in the same order of magnitude as the $\mathrm{CD}[>\mathrm{IP}]$, the absolute values are all smaller and, in particular, differ in their sign for the two- and four-photon excitations, see Table 5. There are several possible reasons for these discrepancies.

First of all, the chosen escape distance of $d=100 a_{0}$ is relatively large (compared to values used by others ${ }^{28}$ ), and results in rather low decay rates ranging from $0.19 /$ fs to $0.29 /$ fs for electronic states above the IP $(11.81 \mathrm{eV})$. For comparison, the laser frequency for the resonant $S_{0} \rightarrow S_{2}$ excitation is with $11 /$ fs about fifty times higher. The large $d$-value was, however, necessary as small values cause the $\left|\mathrm{CD}_{\text {ion }}\right|$ value to diminish. Apparently, interim interactions even with states above the IP are important at these laser pulse conditions, such that any transient population is quasi instantaneously destroyed during the action of the field if the decay rates are too high. Second, the field strengths had to be further increased, as otherwise the $\mathrm{CD}_{\text {ion }}$ values came out two to up to three orders of magnitude smaller than the corresponding $\mathrm{CD}[>\mathrm{IP}]$ values. This may again be attributed to the weakened interactions with the decaying states above the IP. However, high field strengths usually result

Table 5 Comparison of the $\mathrm{CD}\left[\mathrm{S}_{2}\right], \mathrm{CD}[>\mathrm{IP}]$ and $\mathrm{CD}_{\mathrm{ion}}$, as obtained from TD-CIS(D), for excitations with either $\omega_{02}, \omega_{02} / 2$ or $\omega_{02} / 4$, incorporating electric and magnetic dipole as well as electric quadrupole interactions, in an ensemble of rotationally averaged $(R)-P O$ molecules. Field strengths, given in parentheses, have been adapted for $C D_{\text {ion }}$, see text for details. For other parameters see Table 1

\begin{tabular}{lccc}
\hline & $\mathrm{CD}\left[\mathrm{S}_{2}\right][\%]$ & $\mathrm{CD}[>\mathrm{IP}][\%]$ & $\mathrm{CD}_{\text {ion }}[\%]$ \\
\hline$\omega_{02}$ & 2.2 & 2.0 & $1.4\left(1 \mathrm{GV} \mathrm{m}^{-1}\right)$ \\
$\omega_{02} / 2$ & -1.2 & -0.51 & $0.22\left(5 \mathrm{GV} \mathrm{m}^{-1}\right)$ \\
$\omega_{02} / 4$ & 0.32 & 0.19 & $-0.16\left(20 \mathrm{GV} \mathrm{m}^{-1}\right)$
\end{tabular}

in less enantiospecific transitions, see Appendix D and ref. 11, and may eventually amplify the transfer of population back to lower states, in particular, for low decay rates.

Therefore, we cannot tell for sure whether $\mathrm{CD}[>\mathrm{IP}]$ or $\mathrm{CD}_{\text {ion }}$ is the better description for the $\mathrm{CD}$ in the ion yield. It is possible that 150 states are still too few to obtain reliable values for $\mathrm{CD}_{\text {ion }}$, or that there are actually too many states above the IP which are accessible by enantiospecific transitions altering the $\mathrm{CD}[>\mathrm{IP}]$ too much. A higher number of states is, however, beyond the scope of this study. Because the $\mathrm{CD}[>\mathrm{IP}]$ produces values which are closer to the model we used in the previous sections, i.e. it seems to better reflect the CD of the states below the IP, we will stick with the $\mathrm{CD}[>\mathrm{IP}]$ to study the $\mathrm{CD}$ as a function of frequency in the next section.

\subsection{Frequency dependence}

Fig. 3 shows the $\mathrm{CD}[>\mathrm{IP}]$ as a function of the excitation wavelength. Except for the wavelength, the pulse parameters are the same as employed for the four-photon excitations, see Table 1, because we study wavelengths between 700 and $930 \mathrm{~nm}$ to cover the range used in experiment. ${ }^{9}$ As our simulations are based on $a b$ initio data which most certainly differ from the real energetics of the molecule for high electronic states, we do not expect to perfectly reproduce the experiment, but to learn more about what determines the CD in the ion yield.

The wavelength dependent $\mathrm{CD}[>\mathrm{IP}]$ values are roughly one order of magnitude smaller than the CD in ion yields found in experiment, which are $(0.4 \pm 0.5) \%$ at $738 \mathrm{~nm},(2.2 \pm 0.9) \%$ at $810 \mathrm{~nm}$ and $(1.9 \pm 1.1) \%$ for $878 \mathrm{~nm} .{ }^{9}$ Furthermore, we find wavelengths that cause positive $\mathrm{CD}$ values and those which cause negative ones. In the experiment only positive values were found for the three investigated wavelengths. Apparently, the $\mathrm{CD}[<\mathrm{IP}]$ reacts very sensitively to the wavelength, as there are strong changes within a few ten nanometers for some cases. If this sensitivity is caused by achieving resonance with a single (or very few) electric excited states of $(R)$-PO, then a population analysis at the middle of the laser pulse $\left(t_{\mathrm{p}} / 2\right)$ and at the end of the laser pulse $\left(t_{\mathrm{p}}\right)$ should reveal it. This is particularly of

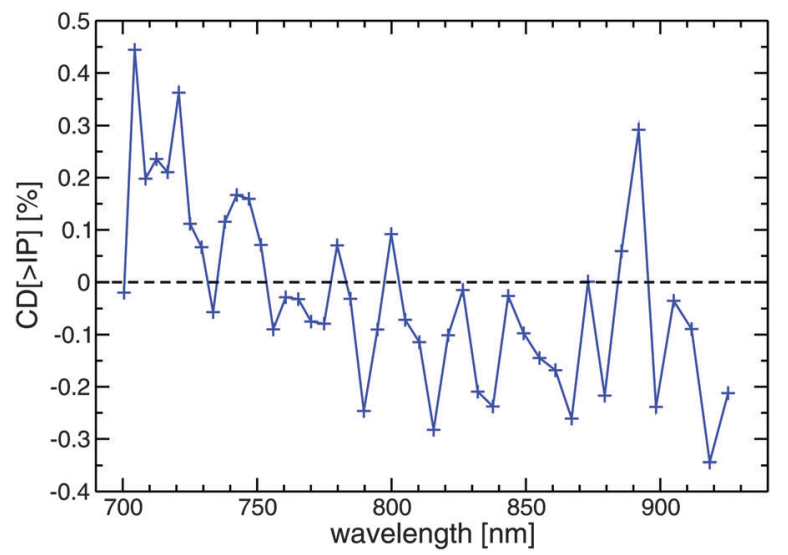

Fig. $3 \mathrm{CD}[>\mathrm{IP}]$ of rotationally averaged $(R)-\mathrm{PO}$ molecules for a selection of excitation wavelengths, as obtained from TD-CIS(D). Other laser pulse parameters are the same as for the four-photon excitation, see Table 1. 
interest, as measurements of the ion yield with respect to the laser pulse energy indicate that three- to seven-photon processes take place for the three investigated wavelengths. ${ }^{9}$ We picked the highest $\mathrm{CD}[>\mathrm{IP}]$ value $(+0.44 \%)$ at $704 \mathrm{~nm}(1.76 \mathrm{eV})$ and the lowest $(-0.34 \%)$ at $918 \mathrm{~nm}(1.35 \mathrm{eV})$, as well as the prominent peak $(+0.29 \%)$ at $892 \mathrm{~nm}(1.39 \mathrm{eV})$ for further analysis:

For $1.76 \mathrm{eV}(704 \mathrm{~nm})$ we find that the full manifold of electronic states are populated at $t_{\mathrm{p}} / 2$. Except for $\mathrm{S}_{0}, \mathrm{~S}_{1}$ is the most populated one below the IP at that moment (followed by $S_{17}$ and others), although its energy is further off resonance than the energy of $S_{2}$ or even $S_{3}$. Here the magnitude of the electric dipole moment seems to be more important than the resonance condition, as it is for $S_{1}$ much larger than for $S_{2}$ or $S_{3}$, see Table 3 . At the end of the laser pulse $\left(t_{\mathrm{p}}\right)$ states $\left(\mathrm{S}_{0},\right) \mathrm{S}_{2}$ and $\mathrm{S}_{3}$ are the most populated states below the IP, which can be interpreted by an initial four-photon transition $(4 \times 1.76 \mathrm{eV}=$ $7.04 \mathrm{eV}$ ) to $\mathrm{S}_{2}$ and $\mathrm{S}_{3}$, followed by e.g. a three-photon transition to the ionization continuum $(7.04 \mathrm{eV}+3 \times 1.76 \mathrm{eV}>11.81 \mathrm{eV})$. The $\mathrm{CD}\left[\mathrm{S}_{2}\right]$ value for the four-photon transition to $\mathrm{S}_{2}$ is positive and about two times larger in magnitude compared to the corresponding negative value for $\mathrm{S}_{3}$ (not shown). Although this is consistent with the positive sign of $\mathrm{CD}[>\mathrm{IP}]$ found at $704 \mathrm{~nm}$, it does not explain why the $\mathrm{CD}[>\mathrm{IP}]$ is significantly larger $(0.44 \%)$ than for the exact four-photon excitation to $S_{2}(0.19 \%)$, see Table 5 . However, many more states are additionally populated. For instance, we cannot ignore the fact that states $\mathrm{S}_{38}$ to $\mathrm{S}_{41}$ energetically lie (10.46-10.72 eV) well within the range of a six-photon excitation $(6 \times 1.76 \mathrm{eV}=10.56 \mathrm{eV})$. In addition, there are more states involved which cannot all easily be attributed to an excitation by a integer number of photons. Thus we can conclude that complex excitation pathways take place.

For $1.35 \mathrm{eV}(918 \mathrm{~nm})$ and $1.39 \mathrm{eV}(892 \mathrm{~nm})$ similar observations are made as before: At $t_{\mathrm{p}} / 2$ mainly those states with highest absolute electronic transition dipole moments are populated, although many of them are not in resonance, i.e. the molecule is significantly polarized during interaction with the laser pulse. At $t_{\mathrm{p}}$ many states below the IP remain populated and offer clues to which states the electronic wavepacket has passed. Those states with rather high populations may indeed be attributed to resonant multiphoton transitions from the electronic ground state. It is these transitions which dominate the $\mathrm{CD}[>\mathrm{IP}]$ with their dipole and (electric) quadrupole moments. Therefore, we did not find any indication of a single dominant multiphoton transition to or via one particular electronic state. In addition, the observed $\mathrm{CD}[>\mathrm{IP}]$ could not be derived from the one-photon $g$-values of the dominant transitions.

Although our analyses indicate that resonant multiphoton excitations via several specific electronic states determine the $\mathrm{CD}[>\mathrm{IP}]$, the process is still more complex, as we only analysed the most prominent excitation pathways. Actually many more states are involved. One must also keep in mind that in our model even the transitions from intermediate states below the IP to those above the IP may be enantiospecific, altering the population difference built up in the previous transition.

We also wish to stress again that one-photon $g$-values did not prove to be suitable for the analysis, as they did not always agree with the CD-value of a multiphoton transition to the same excited state, see also Section 3.3. One-photon anisotropy factors are, however, in many cases the only available data for a prediction. ${ }^{9}$ Therefore, a prediction of the CD in the ion yield after non-resonant multiphoton ionization remains challenging.

\section{Conclusions}

In this paper we extended our methodology to calculate the CD in ion yields via laser driven electron wavepacket dynamics by several aspects. Instead of resonant one-photon excitations, also resonant two- and four-photon as well as non-resonant multiphoton excitations were treated. Due to the two- and higher photon transitions we introduced electric quadrupole interactions. As for non-resonant excitations no target electronic state can uniquely be determined, the CD had to be calculated from the population of electronic states above the ionization potential whose lifetimes were either infinite or finite. These extensions allowed for the simulation of the experimental CD in ion yields as a function of the fs-laser pulse frequency for the non-resonant multiphoton ionization of $(R)$-propylene oxide.

Several findings were reported. For one-photon transitions is was shown that the rotatory strength is not a good quantity for estimating the distinction of enantiomers in the ion yield. In fact, the anisotropy factor is more reliable in this sense, because it accounts for the fact that the CD value is normalized by the sum of populations (or ion yields). However, these findings apply strictly speaking only for a two-level system in the weak field limit. For increasing field strengths, the CD value decreases, namely the faster the higher the respective electric transition dipole moments. While for one-photon transitions the electric quadrupole interactions do not contribute to the CD at all, they become of importance for multiphoton excitations, being even capable of changing the sign of the CD-value.

For non-resonant excitations the $\mathrm{CD}$ is, in general, determined by non-linear interactions with the manifold of electronic states which require permanent and transition matrix elements for and between every electronic state. However, electric (transition) dipole moments are usually significantly larger than their magnetic dipole and electric quadrupole analogs. Therefore, they seem to determine which states are polarized most during laser interactions. Then, multiphoton resonance condition determined by the laser frequency mainly control which states remain populated at the end. Eventually the strongest transitions seem to dominate the observed CD. However, since a large variety of transitions take place and none of them are resonant one-photon excitations, the prediction of the CD in ion yield only based on one-photon anisotropy factors appears to be practically impossible. This confirms experimental observations, where the CD in the ion yield after an assumed n-photon excitation with a wavelength of $n \lambda$ could not be assigned to the CD in one-photon absorption at wavelength $\lambda .^{9}$

Conditions that ensure a resonant one-photon excitation from the start (preferably to a state with a high absolute anisotropy factor) seem, hence, the best way to distinguish enantiomers in 
ion yields on the first glance. Still, for one-photon transitions with small magnetic dipole transition moments, two-photon transitions might be an alternative if certain multipole moments (inter alia permanent electric dipole and quadrupole moments) support a good chiral distinction. ${ }^{29}$ Finally, a subsequent enantiospecific excitation of the parent ion after ionization might also result in higher CD values in the ion yields of fragments preferably formed from the electronically excited parent ion. ${ }^{12,29}$

Some of the extensions of our methodology have proven to be limited. In electron wavepacket dynamics we found that electronic states above the ionization potential have an impact on the CD due to transient interactions with these states, i.e. they allow for additional polarization of the molecule by the field. However, these electronic states are not a perfect description of the continuum states of the free electron. Yet, the approach of bound stationary states above the IP seems to work rather well, while rendering these states non-stationary significantly reduced the response of the electronic structure to the field. But it is also clear that simply adding a manifold of quasicontinuum states will not automatically improve the model in terms of the calculation of the $\mathrm{CD}$, in particular, as transition matrix elements are not available by first principle calculations. Although one can always increase the number of electronic states to the basis set limit of the ab initio calculations, if the computational effort can be handled, the awkward character of the quasi-ionized highly excited electronic states will remain. An alternative ansatz could be to apply hybrid basis sets, composed of local Gaussian-type and plane wave basis functions, for the $a b$ initio calculations. If this will improve the description of the CD ion yield remains to be shown.

Despite the incomplete description of the continuum, the presented methodology is applicable to any molecule for which excited state energies and transitions moments are available. They may be obtained by any quantum chemical method, for instance, by density functional theory in case of larger biomolecules.

\section{Appendix A: detailed analysis of UV and ECD spectra}

The simulated UV and ECD spectra are shown in Fig. 2, the corresponding data is given in Table 2 . The positions of the three main peaks (7.0, 7.3 and around $8.2 \mathrm{eV}$ ) of our simulated UV spectrum are all slightly too low in energy compared to the three band maxima found in experiment (7.1, 7.7 and $\left.8.4 \mathrm{eV}^{33}\right)$. These deviations are even more pronounced for CC2 $(6.52,6.92$, 6.97 and $7.13 \mathrm{eV}$ ) on a similar level of theory, but decrease when advancing to CCSD $(7.31,7.55,7.75$ and $7.95 \mathrm{eV}$ ) or SAC-CI $(7.01,7.71$ and $8.44 \mathrm{eV}) \cdot{ }^{34,35}$ In accordance with these theoretical investigations all excited states within this energy range are mainly characterized by transitions into Rydberg-like orbitals starting either from HOMO-1, the non-bonding orbital at the oxygen $\mathrm{n}(\mathrm{O})$, or the HOMO, a $\sigma$-type MO localized along the bonds of the oxirane ring, see Table 2. A clear characterization of the various Rydberg-like orbitals was, however, in many cases very difficult, as s- and p-character often mix. Still, for the two energetically lowest states with high oscillator strength $\left(\mathrm{S}_{1}, \mathrm{~S}_{4}\right)$ we can assign $\mathrm{n}(\mathrm{O}) \rightarrow 3 \mathrm{~s}$ Rydberg and $\mathrm{n}(\mathrm{O}) \rightarrow 3 \mathrm{p}$ Rydberg transitions in agreement with others. ${ }^{33,35}$ However, their energies (6.95 and $7.29 \mathrm{eV}$ ) are by about $0.2-0.4 \mathrm{eV}$ too low compared to the maxima of the vibrationally broadened bands found in experiment (7.1 and 7.7 eV). Moreover, the states $S_{2}, S_{3}$ lie so close to $S_{1}$ such that they cannot clearly be attributed to two of the three expected $\mathrm{n}(\mathrm{O}) \rightarrow 3$ p Rydberg excitations.

For the third broad band that spans from 144 to $157 \mathrm{~nm}$, see Fig. 2, the excited state $S_{11}$ may be picked as reference, as it agrees very well in excitation energy $(8.35 \mathrm{eV}, 149 \mathrm{~nm})$ and the nature of the transition ( $\sigma \rightarrow 3 p$ Rydberg) to experimental $(8.4 \mathrm{eV})$ and theoretical findings of others $(8.44 \mathrm{eV}) .{ }^{33,35}$ We note, however, that $\mathrm{S}_{6}$ is actually the third lowest state of high oscillator strength, but which is again about $0.4 \mathrm{eV}$ too low in energy $(8.01 \mathrm{eV})$ in comparison to the gas phase UV spectrum $(8.4 \mathrm{eV}) .{ }^{33}$ The character of this transition is dominated by a $\sigma \rightarrow 3 \mathrm{~s}^{\prime \prime}$ Rydberg transition, where the Rydberg orbitals also feature a larger amount of diffuse p-type basis functions. Moreover, the following excited states lie well within in the energy range of the broad experimental band around 8.2-8.8 eV.

The characteristics of the simulated ECD spectrum resemble those of the experimental spectrum, ${ }^{32,33}$ in particular if a larger broadening is used, see inset of Fig. 2. Theory and experiment differ, however, with regard to the details: While the first excited state, $\mathrm{S}_{1}$, has a large negative rotatory strength $\left(R_{1}=\right.$ $\left.-17 \times 10^{-40} \mathrm{cgs}\right)$, the second one, $\mathrm{S}_{2}$, which is very close in energy $(+0.06 \mathrm{eV})$, has a large positive one $\left(R_{2}=+12 \times 10^{-40} \mathrm{cgs}\right)$. This state did not make a strong appearance in the UV spectrum, because its oscillator strength is very low. It significantly reduces the peak height of the first band in the ECD spectrum though, causing only a fair agreement with the particular deep (negative) peak found in experiment. Still sign and size of $R_{1}$ agree well with the experimental one $\left(-13 \times 10^{-40} \mathrm{cgs}\right)^{33}$ and even very well with those of CCSD calculations $\left(-17 \times 10^{-40} \mathrm{cgs}\right) .^{34}$ The state $S_{4}$ has a rotatory strength of $+7 \times 10^{-40} \mathrm{cgs}$ which is again close to the experiment $\left(+6 \times 10^{-40} \mathrm{cgs}\right)$ and well within the range of other methods, e.g. $+5 \times 10^{-40}$ for SAC-CI. ${ }^{35}$ The states between $S_{1}$ and $S_{4}$ contribute to the positive band around $171 \mathrm{~nm}$, see inset of Fig. 2. The third broad peak of the UV spectrum basically appears in the ECD spectrum as three peaks of alternating signs, or two peaks of negative sign (at 148 and $156 \mathrm{~nm}$ ) if a larger broadening is allowed, see inset of Fig. 2. The gas phase ECD spectrum shows indeed a very broad band of very low negative height with a vivid fine structure. ${ }^{32,33} \mathrm{We}$ find seven excited states $\left(S_{6}\right.$ to $\left.S_{12}\right)$ with rotatory strengths of different sizes and signs, which are reached by excitations from either the $\mathrm{n}(\mathrm{O})$ or $\sigma$-MO to a variety of Rydberg orbitals of s- and p-type or combinations of them. As such a one-to-one comparison to the experiment is impossible, in particular as we cannot distinguish between electronic states and vibrational modes. The general negative character of this band is reproduced for the most part, although the rotatory strength is on average too large in magnitude compared to the experiment $\left(-4 \times 10^{-40} \mathrm{cgs}\right) .^{33}$ But even SAC-CI, which was shown to provide a good theoretical description for the first two spectral bands, overestimates the 
size of the rotatory strengths for the state that forms the third band $\left(-10 \times 10^{-40} \mathrm{cgs}\right) .^{35}$

\section{Appendix B: optimized minimum geometry of $(R)-\mathrm{PO}$}

Table 6 lists a selection of bond lengths, angles and dihedrals of the minimum energy geometry obtained from a MP2/aug-cc-pVTZ optimization. Note that, MP2 calculations at $6-311++G(2 d, 2 p)$ level of theory or with the density functional B3LYP, using either basis set, yield values which differ very little (less than one percent) to none from the ones listed in Table 6. Good agreement to the equilibrium structures calculated by similar and more sophisticated methods is found as well. ${ }^{39}$

\section{Appendix C: IR and VCD spectra of $(R)-\mathrm{PO}$}

IR and VCD spectra are obtained from a normal mode analysis of the minimum energy geometry at B3LYP/aug-cc-pVTZ level of theory. The molar absorption coefficient, $\varepsilon$, and its difference between left and right circularly polarized light, $\Delta \varepsilon$, are calculated as described in the supporting information of ref. 11. The simulated spectra (unscaled harmonic frequencies) are shown in Fig. 4 using a Lorentzian broadening of $15 \mathrm{~cm}^{-1}$. Overall a good qualitative agreement to the experimental IR and VCD

Table 6 Characteristic geometric parameters of the minimum energy geometry of $(R)$-propylene oxide as obtained from MP2/aug-cc-pVTZ. For numbering see Fig. 1. For comparison parameters of the semiexperimental equilibrium structure of ref. 39 are given

\begin{tabular}{lll}
\hline Parameter & Size & Ref. 39 \\
\hline$R(\mathrm{C} 1-\mathrm{O})$ & $1.44 \AA$ & $1.43 \AA$ \\
$R(\mathrm{C} 2-\mathrm{O})$ & $1.44 \AA$ & $1.43 \AA$ \\
$R(\mathrm{C} 1-\mathrm{C} 2)$ & $1.46 \AA$ & $1.46 \AA$ \\
$R\left(\mathrm{C} 1-\mathrm{C} 3 \mathrm{H}_{3}\right)$ & $1.50 \AA$ & $1.50 \AA$ \\
$\alpha(\mathrm{C} 1-\mathrm{O}-\mathrm{C} 2)$ & $61.1^{\circ}$ & $61.3^{\circ}$ \\
$\delta(\mathrm{O}-\mathrm{C} 1-\mathrm{C} 2-\mathrm{C} 3)$ & $103.4^{\circ}$ & -
\end{tabular}

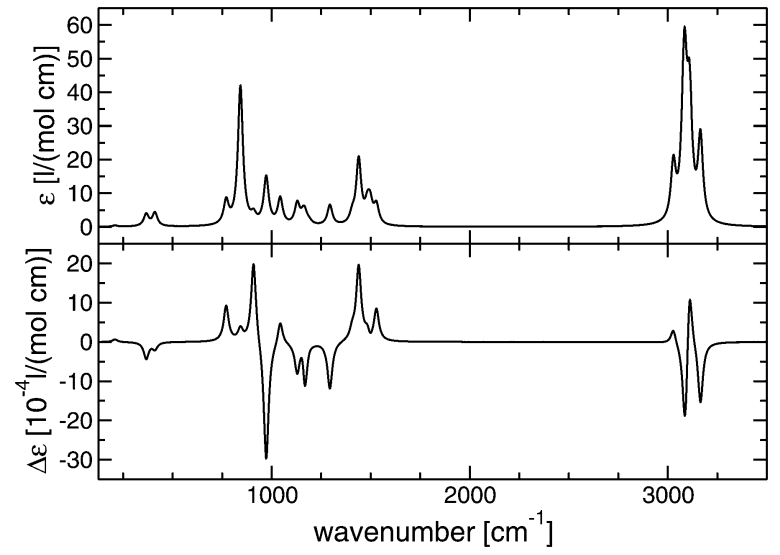

Fig. 4 Simulated IR (top) and VCD (bottom) of (R)-propylene oxide, B3LYP/ aug-cc-pVTZ (unscaled), Lorentzian broadening of $15 \mathrm{~cm}^{-1}$ ( $\hat{=} \mathrm{HWHM}$ ). spectra as well as to first principles calculations is found. ${ }^{39-41}$ Merely, the relative intensities of some of the $\mathrm{CH}$-stretching modes, see peaks around $3100 \mathrm{~cm}^{-1}$, are not perfectly reproduced and they are slightly blue-shifted with respect to the experiment.

\section{Appendix D: correlation between $\mathrm{CD}\left[\mathrm{S}_{j}\right]$ and $g_{j}$}

In Fig. 5 (top) the $\mathrm{CD}\left[\mathrm{S}_{j}\right]$ is plotted as a function of the field strength $\mathcal{E}_{0}$ for selected electronic excited states of $(R)$-PO. In each case the molecule was kept in its optimal initial orientation, as described in Section 2.3, to allow for a best possible comparison with the anisotropy factor $g$. The $\mathrm{CD}\left[\mathrm{S}_{j}\right]$ was calculated for a single orientation after resonant excitation with $\omega=$ $\left(E_{j}-E_{0}\right) / \hbar$. The laser pulse duration $t_{\mathrm{p}}$ was $200 \mathrm{fs}$ in each case.

As can be seen on the right ordinate of the graph, the $\mathrm{CD}\left[\mathrm{S}_{j}\right]$ values at very low field strengths, namely $\mathcal{E}_{0}=0.1 \mathrm{GV} \mathrm{m}^{-1}$, correspond very well to the anisotropy factor $\mathrm{g}_{j}$ of the respective state, $c f$. Table 3 . With increasing field strength, however, the $\mathrm{CD}$ values deviate from their respective $g$-values and approach zero. That is, because the sum of populations $\left(\frac{1}{2}\left(P_{\mathrm{LCP}}+P_{\mathrm{RCP}}\right)\right)$, see eqn (4), increases much faster the difference $\Delta P$ (data not shown). In addition, $\Delta P$ will even decrease once most of the population has been transferred to the target state, see ref. 11 for more details.

In Fig. 5 (bottom) the relative change of the $\mathrm{CD}$ with respect to $\mathcal{E}_{0}=0.1 \mathrm{GV} \mathrm{m}^{-1}$, more precisely $|\Delta \mathrm{CD} / \mathrm{CD}(0.1)|=$ $\left|\left(\mathrm{CD}\left(\mathcal{E}_{0}\right)-\mathrm{CD}(0.1)\right) / \mathrm{CD}(0.1)\right|$, is plotted as a function of $\mathcal{E}_{0}$. The functional form may be approximated by a quadratic function. The largest change is observed for $\mathrm{S}_{1}$ : at $\mathcal{E}_{0}=1 \mathrm{GV} \mathrm{m}^{-1}$ the $\mathrm{CD}$ has lost $60 \%$ of its initial value, i.e. the chiral distinction has significantly decreased. The relative change of the $\mathrm{CD}$ with

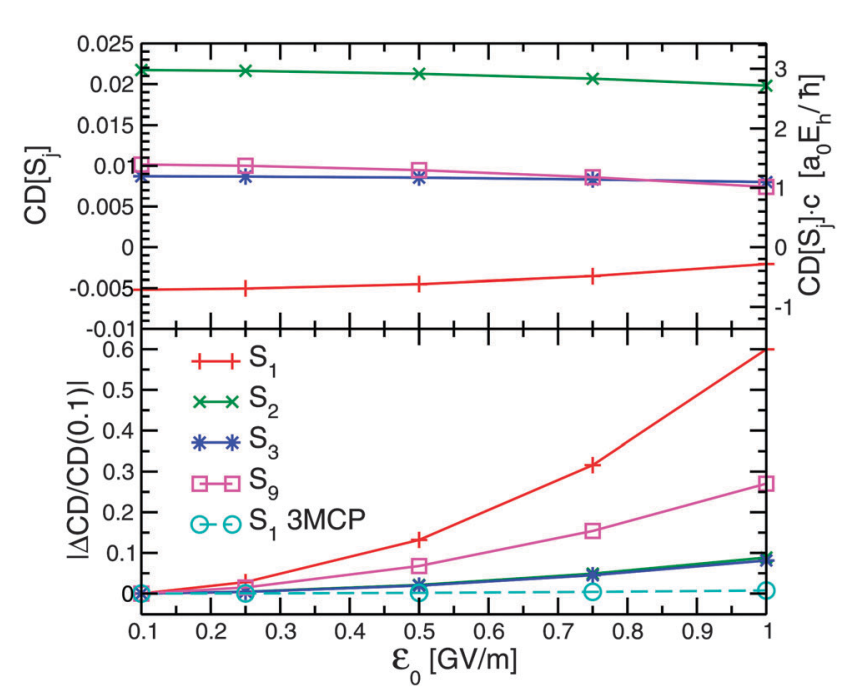

Fig. $5 \mathrm{CD}\left[\mathrm{S}_{j}\right]$ (top) and the absolute value of the relative change of $\mathrm{CD}\left[\mathrm{S}_{j}\right]$ (bottom) as a function of the laser field strength $\mathcal{E}_{0}$ for selected electronic excited states of $(R)$-PO. Right ordinate (top): $C D\left[S_{j}\right]$ scaled by the speed of light (C) for comparison with the anisotropy factor $g$. Data for the $S_{1}$ state of $(R)-3 M C P$ is given for comparison, see circles and dashed lines. 
$\mathcal{E}_{0}$ is dominated by the electric transition dipole moment $\left|\vec{\mu}_{0 j}\right|$, see Table 3 . The higher the $\left|\vec{\mu}_{0 j}\right|$ the larger the relative change of the CD with the laser field strength. Therefore, the anisotropy factor is only a good measure for the CD if field intensity and the electric transition dipole moments in question are rather low. A comparison with the $\mathrm{S}_{1}$ state of $(R)-3 \mathrm{MCP}$ confirms that its large ratio $\frac{\left|\vec{m}_{j 0}\right|}{\left|\vec{\mu}_{0 j}\right|}$ of $19 a_{0} E_{\mathrm{h}} \hbar^{-1}$, see Table 3, ensures a high stability of the $\mathrm{CD}\left[\mathrm{S}_{1}\right]$ with respect to $\mathcal{E}_{0}$, see dashed line in Fig. 5 (bottom).

\section{Appendix E: non-zero CD[1] for an "achiral" two-level system}

While in one-photon excitations the electric quadrupole moment contributions to the circular dichroism vanish for randomly oriented molecules, they play a role in two-photon (or higher) excitations. ${ }^{23}$ Although these contributions are usually small, they can become decisive, in particular if the one-photon $\mathrm{CD}$ is very small or even zero. The later is shown in the following for a two-level model system of states 0 and 1 and parameters given in Table 7. The electric and magnetic transition dipole moments are chosen such that the one-photon $\mathrm{CD}[1]$ is zero, i.e. the $\vec{\mu}_{01}$ is perpendicular to $\Im\left\{\vec{m}_{01}\right\}$ making the system quasi achiral, see Table 7 .

For a two-photon excitation $\left(\omega=0.5 \mathrm{eV} \hbar^{-1}\right)$, the $\mathrm{CD}[1]$ remains zero (i.e. does not change), as long as $\vec{d}=\vec{\mu}_{11}-\vec{\mu}_{00}$ is zero and electric quadrupole interactions are entirely ignored $(\underline{\underline{\hat{Q}}}=\underline{\underline{0}})$, see case (a) in Table 8. A two-photon $\mathrm{CD}[1]$ unequal zero is, however, obtained, if $\vec{d} \neq \overrightarrow{0}$ and as long as $\vec{d}$ is not perpendicular to either $\vec{\mu}_{01}$ or $\Im\left\{\vec{m}_{01}\right\}$, see case (b) in Table 8 . This even holds if quadrupole contributions are excluded. Moreover, if $\vec{d}$ is perpendicular to $\vec{\mu}_{01}$ and $\Im\left\{\vec{m}_{01}\right\}\left(\mu_{z}^{00} \neq 0\right.$, i.e. $\mu_{x}^{00}=\mu_{y}^{00}=0$ ), the electric quadrupole interactions allow for a nonzero two-photon $\mathrm{CD}[1]$, as long as either $\underline{\underline{Q}}_{01}$ (case (c) in Table 8) or $\underline{\underline{\Delta}}=\underline{\underline{Q}}_{11}-\underline{\underline{Q}}_{00}$ (case (d) in Table 8) is nonzero. Therefore, permanent electric dipole moments as well as permanent and transition electric quadrupole moments can result in a nonzero two-photon $\mathrm{CD}$, even if the respective

Table 7 Parameters for the two-level model system. Only non-zero interaction matrix elements are given

\begin{tabular}{ll}
\hline Parameter & Value \\
\hline$E_{1}-E_{0}[\mathrm{eV}]$ & 1 \\
$\mu_{x}{ }^{01}\left[\mathrm{e} a_{0}\right]$ & 1 \\
$\mathfrak{I}_{\left\{m_{y} m^{01}\right\}\left[\mathrm{e} \hbar m_{\mathrm{e}}{ }^{-1}\right]}$ & 1 \\
$\left|\vec{\mu}^{00}\right|\left[\mathrm{e} a_{0}\right]$ & 1 \\
$Q_{y z}{ }^{01}\left[\mathrm{e} a_{0}^{2}\right]$ & -54.4 \\
$Q_{x y}{ }^{00}\left[\mathrm{e} a_{0}^{2}\right]$ & -54.4 \\
$\omega\left[\mathrm{eV} \hbar^{-1}\right]$ & 0.5 \\
$\mathcal{E}_{0}\left[\mathrm{GV} \mathrm{m} \mathrm{m}^{-1}\right]$ & 5.0 \\
$t_{\mathrm{p}}[\mathrm{fs}]$ & 200
\end{tabular}

Table $8 \mathrm{CD}[1]$ for a two-photon $0 \rightarrow 1$ excitations $\left(\omega=0.5 \times\left(E_{1}-E_{0}\right) / \hbar\right)$ in rotational averaged $(N=144)$ model systems, including different electric and magnetic multipole components

\begin{tabular}{llc}
\hline Case & Interactions & $\mathrm{CD}[1][\%]$ \\
\hline (a) & $\underline{\underline{\hat{Q}}}=\underline{\underline{0}},\left|\vec{\mu}^{00}\right|=0$ & 0.00 \\
(b) & $\underline{\underline{\hat{Q}}}=\underline{\underline{0}}, \mu_{x}^{00} \neq 0, \mu_{y}^{00} \neq 0$ & 0.35 \\
(c) & $\underline{\underline{\hat{Q}}}_{01} \neq \underline{\underline{0}}, \underline{\underline{\hat{Q}}}_{00}=\underline{\underline{0}}, \mu_{z}^{00} \neq 0$ & 0.35 \\
(d) & $\underline{\underline{\hat{Q}}}_{01}=\underline{\underline{0}}, \underline{\underline{\hat{Q}}}_{00} \neq \underline{\underline{0}}, \mu_{z}^{00} \neq 0$ & -0.54
\end{tabular}

one-photon $\mathrm{CD}$ is zero. These findings agree with predictions made by Meath and Power based on analytical expressions for Einstein B-coefficients. ${ }^{23}$

\section{Acknowledgements}

The author would like to thank Karl-Michael Weitzel (Marburg), Tillmann Klamroth and Peter Saalfrank for helpful discussions. Financial support by the German Research Foundation (project KR 2942/2) is gratefully acknowledged.

\section{References}

1 R. Judson and H. Rabitz, Phys. Rev. Lett., 1992, 68, 1500-1503.

2 A. Assion, T. Baumert, M. Bergt, T. Brixner, B. Kiefer, V. Seyfried, M. Strehle and G. Gerber, Science, 1998, 282, 919-922.

3 J. Dela Cruz, V. Lozovoy and M. Dantus, J. Phys. Chem. A, 2005, 109, 8447-8450.

4 U. Boesl von Grafenstein and A. Bornschlegl, ChemPhysChem, 2006, 7, 2085-2087.

5 R. Li, R. Sullivan, W. Al-Basheer, R. M. Pagni and R. N. Compton, J. Chem. Phys., 2006, 125, 144304.

6 D. Kröner, M. F. Shibl and L. González, Chem. Phys. Lett., 2003, 372, 242-248.

7 D. Kröner and L. González, Phys. Chem. Chem. Phys., 2003, 5, 3933-3942.

8 H. G. Breunig, G. Urbasch, P. Horsch, J. Cordes, U. Koert and K.-M. Weitzel, ChemPhysChem, 2009, 10, 1199-1202.

9 P. Horsch, G. Urbasch and K.-M. Weitzel, Z. Phys. Chem., 2011, 225, 587-594.

10 P. Horsch, G. Urbasch, K.-M. Weitzel and D. Kröner, Phys. Chem. Chem. Phys., 2011, 13, 2378-2386.

11 D. Kröner, J. Phys. Chem. A, 2011, 115, 14510-14518.

12 P. Horsch, G. Urbasch and K.-M. Weitzel, Chirality, 2012, 24, 684-690.

13 Y. Ma and A. Salam, Chem. Phys., 2006, 324, 367-375.

14 C. Møller and M. S. Plesset, Phys. Rev., 1934, 46, 618-622.

15 T. H. Dunning Jr., J. Chem. Phys., 1989, 90, 1007-1023.

16 R. A. Kendall, T. H. Dunning Jr. and R. J. Harrison, J. Chem. Phys., 1992, 96, 6796-6806.

17 M. Head-Gordon, R. J. Rico, M. Oumi and T. J. Lee, Chem. Phys. Lett., 1994, 219, 21-29. 
18 Gaussian 09 (Rev. A.02) prints out the magnetic transition dipole matrix elements as $\left\langle\Phi_{i}|\vec{r} \times \overrightarrow{\vec{\nabla}}| \Phi_{j}\right\rangle$, i.e. the values have to be multiplied by $1 / 2$ to obtain the employed $\vec{m}_{i j}$ in atomic units $\left(\mathrm{e} \hbar \mathrm{m}_{\mathrm{e}}{ }^{-1}\right)$. Electric transition quadrupole moments $\underline{\underline{Q}}_{0 i}$ between ground and electronic excited state $i$, obtained from the transition density, have to be divided by $\sqrt{2}$. CI-coefficients $D_{a}^{r}$ are multiplied by $\sqrt{2}$ to preserve a norm of one $\left(\sum_{r} \sum_{a}\left|D_{a}^{r}\right|^{2}\right.$ should be one).

19 K. B. Wiberg, C. M. Hadad, T. J. LePage, C. M. Breneman and M. J. Frisch, J. Phys. Chem., 1992, 96, 671-679.

20 M. J. Frisch, G. W. Trucks, H. B. Schlegel, G. E. Scuseria, M. A. Robb, J. R. Cheeseman, G. Scalmani, V. Barone, B. Mennucci, G. A. Petersson, H. Nakatsuji, M. Caricato, X. Li, H. P. Hratchian, A. F. Izmaylov, J. Bloino, G. Zheng, J. L. Sonnenberg, M. Hada, M. Ehara, K. Toyota, R. Fukuda, J. Hasegawa, M. Ishida, T. Nakajima, Y. Honda, O. Kitao, H. Nakai, T. Vreven, J. J. A. Montgomery, J. E. Peralta, F. Ogliaro, M. Bearpark, J. J. Heyd, E. Brothers, K. N. Kudin, V. N. Staroverov, R. Kobayashi, J. Normand, K. Raghavachari, A. Rendell, J. C. Burant, S. S. Iyengar, J. Tomasi, M. Cossi, N. Rega, J. M. Millam, M. Klene, J. E. Knox, J. B. Cross, V. Bakken, C. Adamo, J. Jaramillo, R. Gomperts, R. E. Stratmann, O. Yazyev, A. J. Austin, R. Cammi, C. Pomelli, J. W. Ochterski, R. L. Martin, K. Morokuma, V. G. Zakrzewski, G. A. Voth, P. Salvador, J. J. Dannenberg, S. Dapprich, A. D. Daniels, O. Farkas, J. B. Foresman, J. V. Ortiz, J. Cioslowski and D. J. Fox, Gaussian 09, Revision A.02, Gaussian Inc., Wallingford, CT, 2009.

21 T. Klamroth, Phys. Rev. B: Condens. Matter Mater. Phys., 2003, 68, 245421.

22 P. Krause and T. Klamroth, J. Chem. Phys., 2008, 128, 234307.

23 W. J. Meath and E. A. Power, J. Mod. Opt., 1989, 36, 977-1002. 24 W. J. Meath and A. E. Kondo, J. Mol. Struct.: THEOCHEM, 1991, 232, 23-42.
25 K. Watanabe, T. Nakayama and J. Mottl, J. Quant. Spectrosc. Radiat. Transfer, 1962, 2, 369-382.

26 L. Keldysh, Sov. Phys. JETP, 1965, 20, 1307-1314.

27 E. A. Power and T. Thirunamachandran, J. Chem. Phys., 1974, 60, 3695-3701.

28 S. Klinkusch, P. Saalfrank and T. Klamroth, J. Chem. Phys., 2009, 131, 114304.

29 C. Logé and U. Boesl, Phys. Chem. Chem. Phys., 2012, 14, 11981-11989.

30 L. Greenman, P. J. Ho, E. Karmarchik, D. A. Mazzoni and R. Santra, Phys. Rev. A: At., Mol., Opt. Phys., 2010, 82, 023406.

31 P. Krause, J. A. Sonk and H. B. Schlegel, J. Chem. Phys., 2014, 140, 174113.

32 M. Carnell, S. D. Peyerimhoff, A. Breest, K. H. Gödderz, P. Ochmann and J. Hormes, Chem. Phys. Lett., 1991, 180, 477-481.

33 A. Breest, P. Ochmann, F. Pulm, K. H. Gödderz, M. Carnell and J. Hormes, Mol. Phys., 1994, 82, 539-551.

34 J. Kongsted, T. B. Pedersen, M. Strange, A. Osted, A. E. Hansen, K. V. Mikkelsen, F. Pawlowski, P. Jørgensen and C. Hättig, Chem. Phys. Lett., 2005, 401, 385-392.

35 T. Miyahara, J. Hasegawa and H. Nakatsuji, Bull. Chem. Soc. Jpn., 2009, 82, 1215-1226.

36 S. Góbi and G. Magyarfalvi, Phys. Chem. Chem. Phys., 2011, 13, 16130-16133.

37 Y. Ma and A. Salam, Chem. Phys. Lett., 2006, 431, 247-252. 38 J. A. Schellman, Chem. Rev., 1975, 75, 323-331.

39 V. Barone, M. Biczysko, J. Bloino and C. Puzzarini, J. Chem. Phys., 2014, 141, 034107.

40 P. Chu, F. Guenther, G. Rhoderick and W. Lafferty, in NIST Chemistry WebBook, NIST Standard Reference Database Number 79: Quantitative Infrared Database, ed. P. Linstrom and W. Mallard, National Institute of Standards and Technology, Gaithersburg, MD, 20899, 2014.

41 P. L. Polavarapu, B. A. Hess Jr. and L. J. Schaad, J. Chem. Phys., 1985, 82, 1705-1710. 